\title{
ALMA OBSERVATIONS OF $\rho$-Oph 102: GRAIN GROWTH AND MOLECULAR GAS IN THE DISK AROUND A YOUNG BROWN DWARF
}

\author{
L. Ricci ${ }^{1}$, L. Testi ${ }^{2,3}$, A. NATta ${ }^{3,4}$, A. Scholz ${ }^{4}$, And I. DE Gregorio-Monsalvo ${ }^{5}$ \\ ${ }^{1}$ Department of Astronomy, California Institute of Technology, MC 249-17, Pasadena, CA 91125, USA; 1ricci@astro.caltech.edu \\ ${ }^{2}$ European Southern Observatory, Karl-Schwarzschild-Strasse 2, D-85748 Garching, Germany \\ ${ }^{3}$ INAF-Osservatorio Astrofisico di Arcetri, Largo E. Fermi 5, I-50125 Firenze, Italy \\ ${ }^{4}$ School of Cosmic Physics, Dublin Institute for Advanced Studies, 31 Fitzwilliam Place, Dublin 2, Ireland \\ ${ }^{5}$ Joint ALMA Observatory (JAO)/ESO, Alonso de Cordova 3107, Vitacura 763 0335, Santiago de Chile, Chile \\ Received 2012 October 10; accepted 2012 October 28; published 2012 November 30
}

\begin{abstract}
We present ALMA continuum and spectral line observations of the young brown dwarf $\rho$-Oph 102 at about $0.89 \mathrm{~mm}$ and $3.2 \mathrm{~mm}$. We detect dust emission from the disk at these wavelengths and derive an upper limit on the radius of the dusty disk of $\sim 40 \mathrm{AU}$. The derived variation of the dust opacity with frequency in the millimeter (mm) provides evidence for the presence of mm-sized grains in the disk's outer regions. This result demonstrates that $\mathrm{mm}$-sized grains are found even in the low-density environments of brown dwarf disks and challenges our current understanding of dust evolution in disks. The $\mathrm{CO}$ map at $345 \mathrm{GHz}$ clearly reveals molecular gas emission at the location of the brown dwarf, indicating a gas-rich disk as typically found for disks surrounding young pre-mainsequence stars. We derive a disk mass of $\sim 0.3 \%-1 \%$ of the mass of the central brown dwarf, similar to the typical values found for disks around more massive young stars.
\end{abstract}

Key words: brown dwarfs - circumstellar matter - planets and satellites: formation - stars: individual ( $\rho$-Oph 102) - submillimeter: stars

Online-only material: color figures

\section{INTRODUCTION}

Brown dwarfs (BDs) are very low mass $\left(M \leqslant 0.085 M_{\odot}\right)$, stellar-like objects unable to burn hydrogen. It is now established that young BDs are surrounded by dusty circumstellar disks (Natta \& Testi 2001; Natta et al. 2002; Testi et al. 2002; Scholz \& Jayawardhana 2008) and undergo a T Tauri-like phase during their early evolution that involves disk accretion and outflows (Jayawardhana et al. 2003; Natta et al. 2004).

Because of their very low masses, disks around BDs also represent an extreme environment in which to test planet formation theories. There are a few wide-orbit, giant planets known as companions to BDs, most likely formed as a result of the fragmentation of the proto-BD core or by gravitational instabilities in the outer disk (e.g., 2M1207b, 2M0441b, Chauvin et al. 2005; Lodato et al. 2005; Todorov et al. 2010). However, most extrasolar planets (and all rocky planets) are thought to form via the core accretion process (Matsuo et al. 2007), and evidence of the first steps of this process has been found in disks around more massive pre-main-sequence (PMS) stars. In particular, submillimeter (sub-mm) observations, which probe the bulk of the disk's cold material, have shown that the grains have grown to reach millimeter and centimeter sizes (e.g., Beckwith \& Sargent 1991; Rodmann et al. 2006; Ricci et al. 2010a; Ubach et al. 2012).

In BD disks, infrared spectroscopy has shown that in several sources silicates on the disk surface have been significantly modified in size and crystallinity, as in PMS stars (Sterzik et al. 2004; Apai et al. 2005). Until now, due to the limited sensitivity of sub-mm telescopes, the detection of long wavelength emission from young BDs has been limited to continuum photometry of the few brightest objects (e.g., Scholz et al. 2006; Mohanty et al. 2012). The start of ALMA operations allows us to perform more detailed studies. Here, we report on multi-wavelength continuum and $\mathrm{CO}(J=3-2)$ observations of the young $\mathrm{BD} \rho$-Oph 102 . This object ( $\sim 60 M_{\text {Jup }}$, M6 spectral type) in the $\rho$-Ophiuchi star-forming region (SFR) is known from infrared observations to be surrounded by a disk to have a significant mass accretion rate, and to drive a wind and molecular outflow (Bontemps et al. 2001; Natta et al. 2002, 2004; Whelan et al. 2005; Phan-Bao et al. 2008). In this Letter we report on our ALMA observations, which were designed to derive a solid estimate of the millimeter $(\mathrm{mm})$ spectral index to constrain the dust properties. In particular, we found evidence for grain growth to $\mathrm{mm}$ grain sizes in the outer regions of the $\rho$-Oph 102 disk. Also, we clearly detected molecular gas emission from the disk and derived an estimate for its mass.

\section{ALMA OBSERVATIONS AND RESULTS}

\subsection{Observations and Data Reduction}

We observed $\rho$-Oph 102 using ALMA Early Science in Cycle 0 at Bands 7 and 3 (about 345 and $100 \mathrm{GHz}$, respectively). Observations in Band 7 were performed using 15 antennas in the compact array configuration on 2011 November 3 , and 16 antennas in the extended array configuration on 2012 May 23 (projected baseline lengths in the range from the shadowing limit to $\sim 400 \mathrm{~m}$ ). Observations in Band 3 were conducted with 16 antennas in the extended configuration on 2012 May 8 (projected baseline lengths from $\sim 35$ to $\sim 402 \mathrm{~m}$ ). All observations were done in good and stable weather conditions with precipitable water vapor of $\sim 0.8-1.2 \mathrm{~mm}$ and $\sim 1.9 \mathrm{~mm}$ at Bands 7 and 3, respectively. The ALMA correlator was set to record dual polarization with four separate spectral windows, each providing a bandwidth of $1.875 \mathrm{GHz}$ with channels of $0.488 \mathrm{MHz}$ width. Spectral windows were centered at 331.103, 

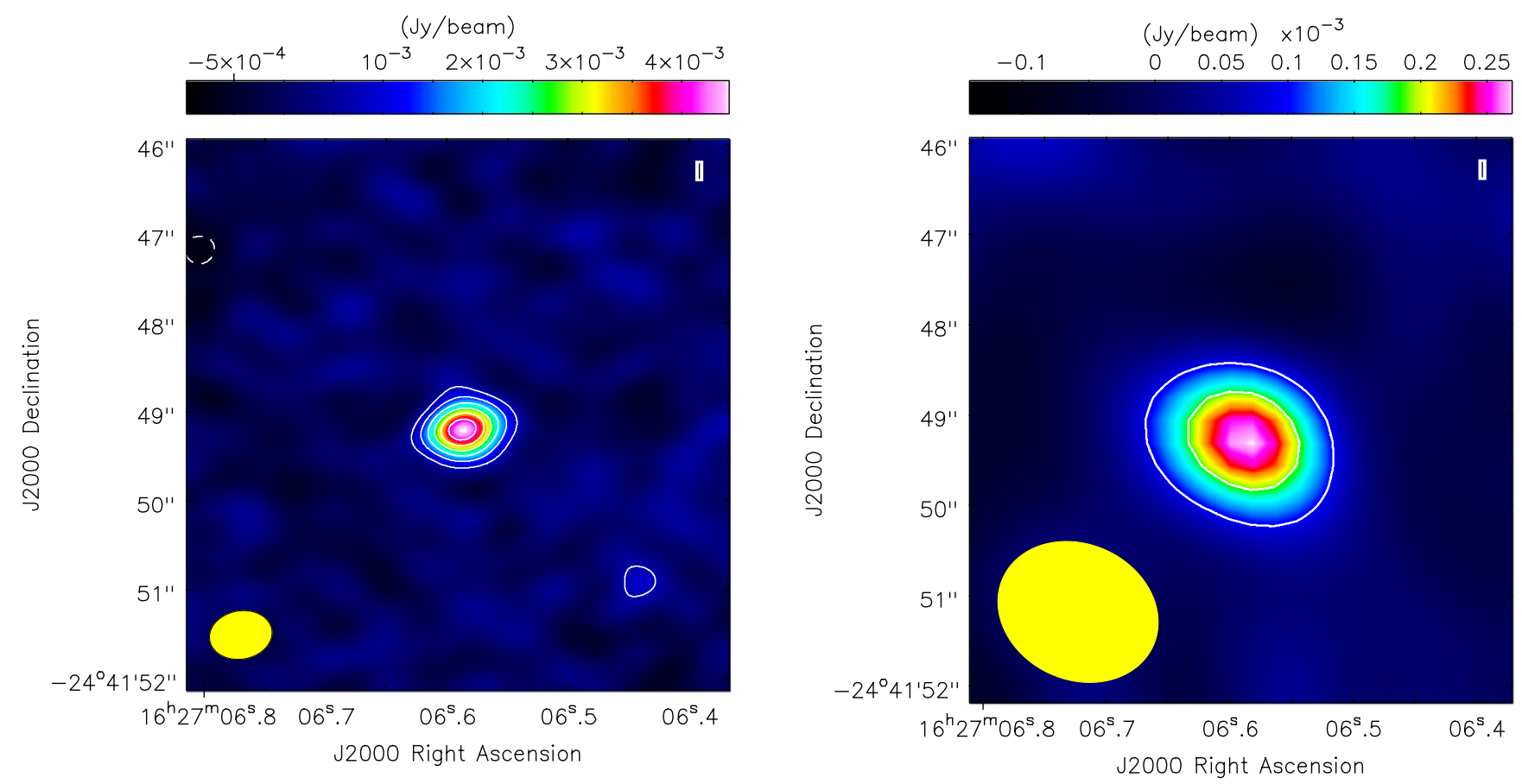

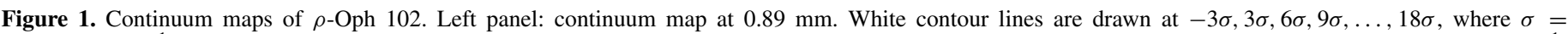

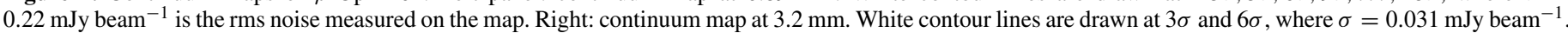

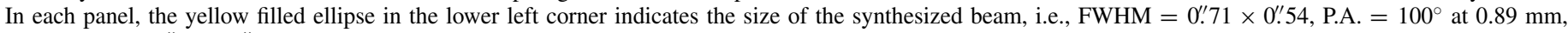

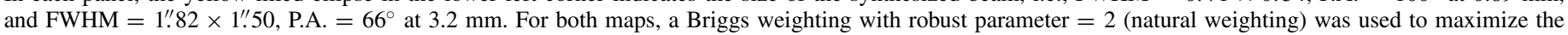
signal-to-noise ratio.

(A color version of this figure is available in the online journal.)

332.998, 343.103, and 344.998 GHz for Band 7 observations, and 86.1021, 87.9976, 98.103, and 99.999 GHz for Band 3. The total integration times on $\rho$-Oph 102 were approximately 15 and 30 minutes in Bands 7 and 3, respectively.

The interferometric visibility data were reduced using the CASA package (McMullin et al. 2007). J1924-292 and 3C279 were observed as bandpass calibrators, Neptune and Titan for flux calibration. Simultaneous observations of the $183 \mathrm{GHz}$ water line with the water vapor radiometers were used to reduce atmospheric phase noise before using J1625-254 for standard complex gains calibration. The flux scale was tied to the Butler-JPL-Horizons 2010 models of Neptune and Titan, resulting in an accuracy of $\sim 10 \%$.

Imaging of the calibrated visibilities was done in CASA. For each band, a continuum map was produced using natural weighting and combining all the channels without line emission. The only line emission detected was the $\mathrm{CO}(J=3-2)$ rotational line in Band 7, which was imaged separately. The $\mathrm{CO}(3-2)$ shows significant extended emission as well as compact structure. We imaged the $\mathrm{CO}$ data in two separate ways to highlight the different components: we first used all the data, natural weighting, and a Gaussian taper of the visibilities to recover as much as possible of the extended emission and compare with the low angular resolution map of Phan-Bao et al. (2008); additionally, we also imaged the data from baselines exceeding $\sim 60$ $\mathrm{k} \lambda$ with robust weighting to filter out emission on scales larger than about $3^{\prime \prime}$, or $\sim 400 \mathrm{AU}$ at the Ophiuchus distance, and we highlighted the compact emission associated with $\rho$-Oph 102.

\subsection{Continuum Maps}

Figure 1 shows the continuum maps at about $0.89 \mathrm{~mm}$ (Band 7) and $3.2 \mathrm{~mm}$ (Band 3). Dust emission from the disk surrounding $\rho$-Oph 102 is clearly detected in both maps. We measured flux densities of $4.10 \pm 0.22 \mathrm{mJy}$ at $0.89 \mathrm{~mm}$ and of $0.22 \pm 0.03 \mathrm{mJy}$ at $3.2 \mathrm{~mm}$. The obtained rms noise levels are consistent with the theoretical expectations given the onsource integration times. This gives a sub-mm spectral index $\alpha\left(F_{v} \propto v^{\alpha}\right)$ between 0.89 and $3.2 \mathrm{~mm}$ of $\alpha_{0.89-3.2 \mathrm{~mm}}=$ $2.29 \pm 0.16$, where the uncertainty accounts for both the flux calibration and the rms noise in the maps. This value lies well within the range of values measured for disks around single PMS stars in the $\rho$-Oph and Taurus SFRs (see Figure 2). This suggests that the physical properties of the dust grains emitting in the sub-mm, e.g., their size, do not significantly vary in disks around PMS stars and BDs. The implications of this result will be discussed in Section 3.

Using observations with the Submillimeter Array (SMA; Ho et al. 2004), Phan-Bao et al. (2008) reported a low signal-tonoise flux density of $7 \pm 3 \mathrm{mJy}$ at about $1.3 \mathrm{~mm}$. Interpolating between our two measurements, we expect a flux density of $1.7 \pm 0.3 \mathrm{mJy}$ at $1.3 \mathrm{~mm}$, lower than the value previously reported, although the discrepancy is only at the $2 \sigma$ level. Note that already with $\sim 15$ minutes on-source with ALMA in Early Science we reached a sensitivity $\sim 10$ times better than SMA.

When inspecting the visibility data, within the uncertainties we did not find evidence for a decrease of the visibility amplitude with projected baseline length, either at the shorter or at the longer baseline lengths probed by our observations. We conclude that our observations did not spatially resolve the disk continuum emission. Considering our angular resolution of $\sim 0$.' 6 at $0.89 \mathrm{~mm}$ and a distance of $130 \mathrm{pc}$ for the $\rho$-Oph SFR (Wilking et al. 2008; Lombardi et al. 2008), this indicates that the dust is concentrated within less than $\sim 40 \mathrm{AU}$ from the central BD. 


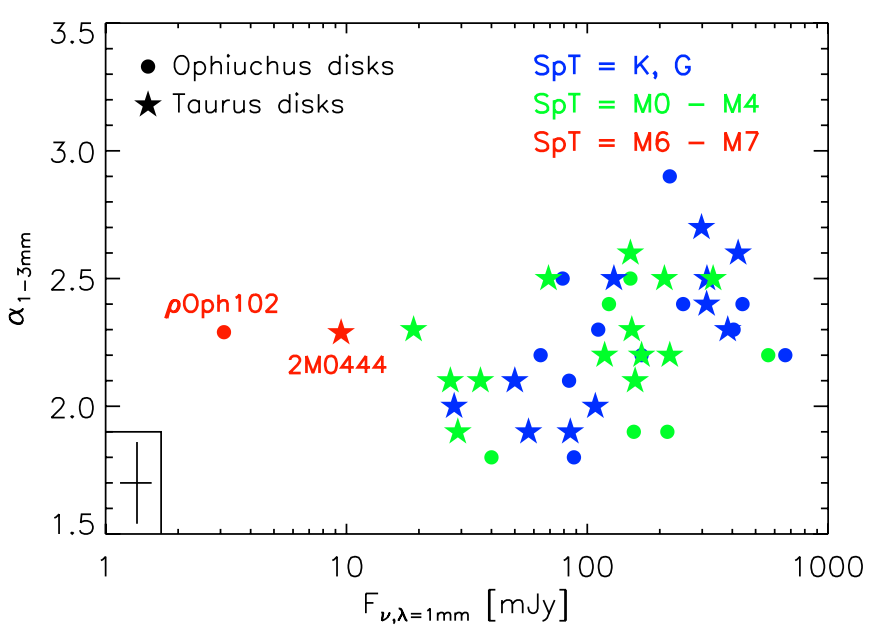

Figure 2. Flux at $1 \mathrm{~mm}$ vs. spectral index between 1 and $3 \mathrm{~mm}$ for disks around single PMS stars and brown dwarfs. Different colors and symbols refer to different stellar/sub-stellar spectral types and regions as indicated in the plot. Data for Taurus disks are from Ricci et al. (2010a, 2012), for Ophiuchus disks from Ricci et al. (2010b), for 2M0444 from Bouy et al. (2008) and L. Ricci et al. (2012, in preparation), and for $\rho$-Oph 102 from this Letter. Note that for same disks the values of the $1 \mathrm{~mm}$ flux density have been derived by interpolating between nearby wavelengths. The typical uncertainties of the data are shown in the lower left corner.

(A color version of this figure is available in the online journal.)

\subsection{CO Molecular Gas Map}

The low-angular-resolution, tapered natural weighting map shows a morphology of the $\mathrm{CO}(J=3-2)$ that closely resembles the map of the $\mathrm{CO}(J=2-1)$ emission presented by Phan-Bao et al. (2008). We confirm the same main emission features as observed in the SMA map. They interpreted these structures as a bipolar molecular outflow emitted by $\rho$-Oph 102 . Even if the signal-to-noise ratio in our map is significantly better than in the SMA data, our data are most likely significantly affected by missing flux at short baselines. Furthermore, the most prominent features attributed to the outflow are close to the edge of the ALMA primary beam, making it difficult to accurately determine the emission and its extent. For these reasons, we will not discuss the extended emission and outflow further in this Letter.

In the $\operatorname{CO}(J=3-2)$ low-angular-resolution map, we also detected compact emission coincident with the continuum emission described in Section 2.2. The left panel of Figure 3 shows the map of integrated $\mathrm{CO}(J=3-2)$ emission, obtained by filtering out the extended $\mathrm{CO}$ emission as described at the end of Section 2.1. Compact emission, which we associate with $\rho$-Oph 102 , is clearly detected in several channels. The disk is detected at a $>10 \sigma$ level, with a velocity-integrated total flux of about $530 \pm 45 \mathrm{mJy} \mathrm{km} \mathrm{s}^{-1}$. The right panel of Figure 3 shows the intensity-weighted velocity field of $\mathrm{CO}$ molecular gas. We tentatively find the presence of a velocity gradient along the disk, spanning a range of radial velocities of $\approx 1 \mathrm{~km} \mathrm{~s}^{-1}$. This is consistent with gas in Keplerian rotation in an inclined disk orbiting at distances $\gtrsim 10 \mathrm{AU}$ from a $0.06 M_{\odot} \mathrm{BD}$. However, the angular $\left(\sim 0^{\prime \prime} .5\right)$ and velocity $\left(\sim 0.4 \mathrm{~km} \mathrm{~s}^{-1}\right)$ resolutions of our observations do not allow a full characterization of the rotation curve of the disk, and non-Keplerian rotation curves are still possible.

\section{DISCUSSION}

To derive the disk mass and physical properties of the grains, we model the mm continuum emission using a twolayer (surface+midplane) model of a flared disk heated by the radiation of the central BD (Chiang \& Goldreich 1997; Dullemond et al. 2001).

The adopted sub-stellar photospheric properties of $\rho$-Oph 102 and the dust model are described in the caption of Figure 4.
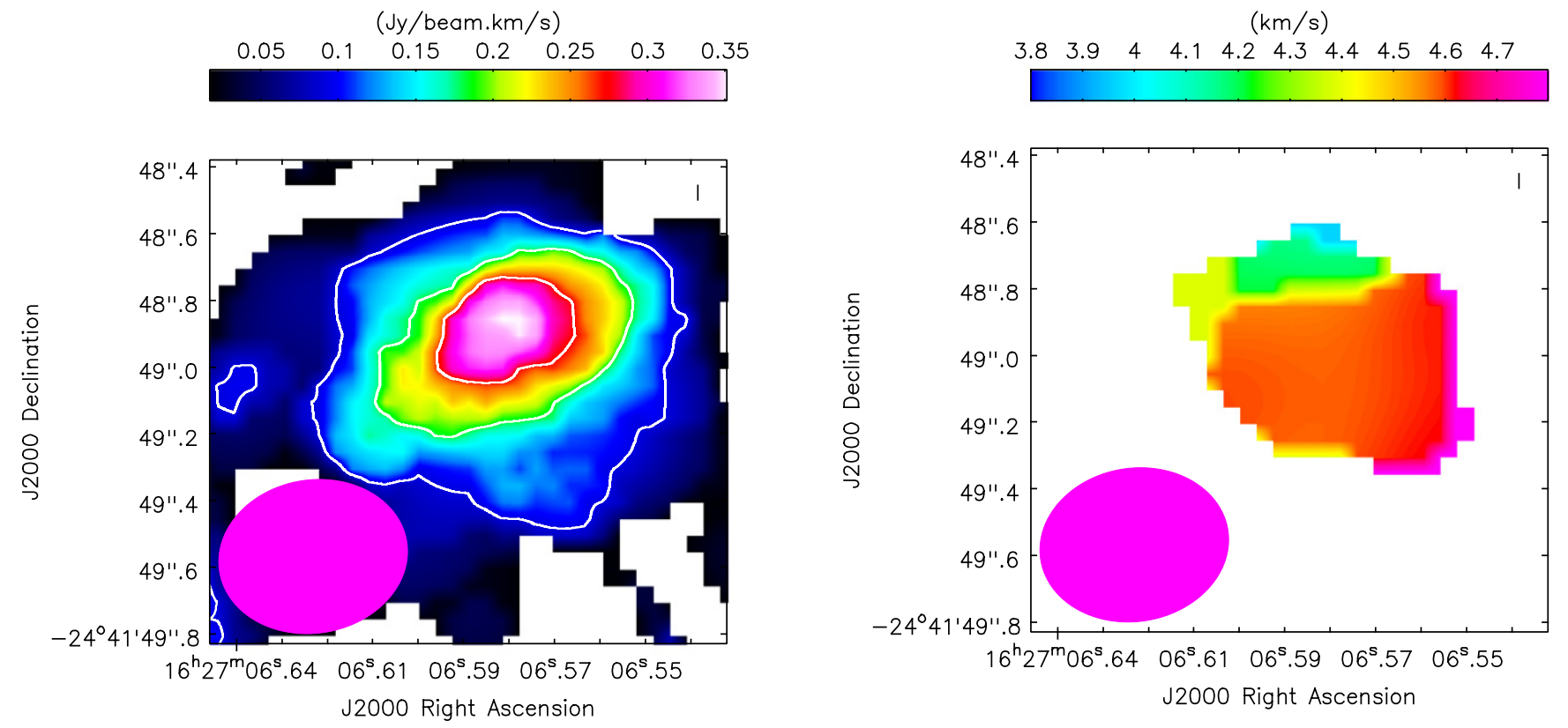

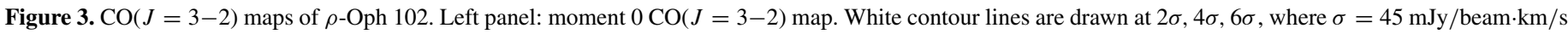

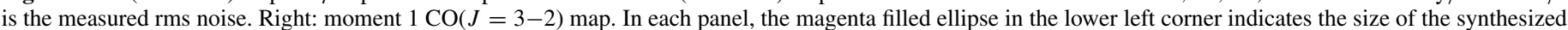

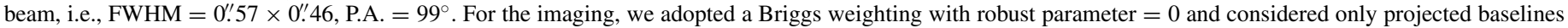

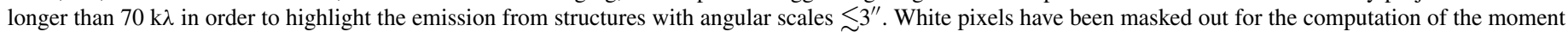
maps.

(A color version of this figure is available in the online journal.) 


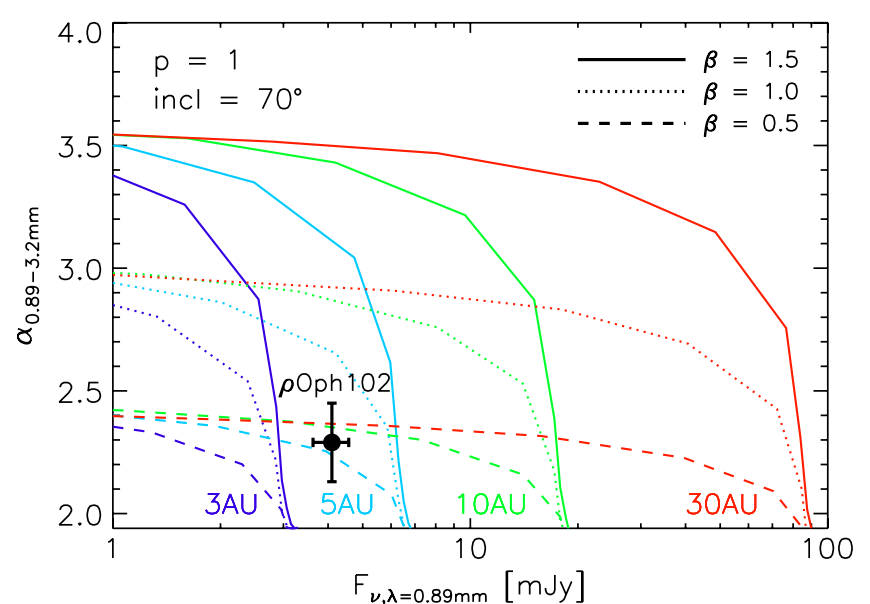

Figure 4. Submillimeter flux density vs. spectral index for disk models for $\rho$-Oph 102. The black dot shows our ALMA data for $\rho$-Oph 102. Each line represents the prediction of disk models with the same disk outer radius and dust opacity spectral index $\beta$, but increasing disk mass from left to right. The values considered for the disk radii and $\beta$ are indicated. Each model was computed by assuming a radial profile of the surface density $\Sigma \propto r^{-p}$, with $p=1$, and a disk inclination of $70^{\circ}$. For the sub-stellar properties of $\rho$-Oph 102, we took a mass $M_{\star}=0.06 M_{\odot}$, luminosity $L_{\star}=0.08 L_{\odot}$, and effective temperature $T_{\text {eff }}=2700 \mathrm{~K}$ (from Natta et al. 2002). For the computation of the dust opacities, we considered porous spherical grains made of astronomical silicates, carbonaceous materials, and water ice (optical constants for individual components from Weingartner \& Draine 2001; Zubko et al. 1996; Warren 1984, respectively) and adopted a simplified version of the fractional abundances used by Pollack et al. (1994), as done in Ricci et al. (2010a, 2010b). Grain sizes are distributed as a power law $n(a) \propto a^{-q}$ with $q=3.0$ and between a minimum grain size of $0.1 \mu \mathrm{m}$ and a maximum grain size which determines the value of $\beta$ (for more details, see Ricci et al. 2010a).

(A color version of this figure is available in the online journal.)

Since our observations did not allow us to spatially resolve the dust emission of the disk, we could not strongly constrain the disk radius and inclination. Our analysis of the unresolved disk photometry follows the procedure outlined in Testi et al. (2001), adapted for the case of young BDs. For the disk surface density, we adopted a radial profile $\Sigma \propto r^{-p}$ with $p=1$, which is the typical value found for $\mathrm{T}$ Tauri disks in the submm (e.g., Isella et al. 2009; Andrews et al. 2010; Guilloteau et al. 2011). A large value of the disk inclination is suggested by the detection of an optical jet (Whelan et al. 2005) and by the nearly symmetric morphology of the bipolar outflow in molecular CO (Phan-Bao et al. 2008). At the same time, the visual extinction of the $\rho$-Oph 102 photosphere, i.e., $A_{V} \approx 3$ (Natta et al. 2002) poses an upper limit of $\sim 80^{\circ}$ for the disk inclination, as a more inclined disk would absorb the (sub)stellar radiation much more efficiently (see Skemer et al. 2011). In the following we assume an inclination of $70^{\circ}$; however, our results do not depend critically on this choice.

\subsection{Disk Mass and Size}

The main results of our modeling can be derived from Figure 4, which shows the ALMA data overlaid with the predictions from the disk models presented above. Disk models with radii larger than $\gtrsim 5$ AU can reproduce the data only if the spectral index of the dust opacity $\beta\left(\kappa_{v} \propto v^{\beta}\right)$ is smaller than 1. This is because for these disks the dust emission is predominantly coming from the optically thin outer regions of the disk. In this case, the spectral index of the spectral energy distribution reflects the dust opacity spectral index, and a relatively low value of $\beta$ is needed to explain the measured spectral index $\alpha_{0.89-3.2 \mathrm{~mm}}$. Models with disk radii $<5 \mathrm{AU}$ reproduce the observed continuum flux and spectral index with a broad range of $\beta$-values. This is because very small disks have to be relatively dense to explain the observed sub-mm fluxes: the dust emission in these models becomes mostly optically thick and, in this regime, it does not depend on the dust opacity law (cf. the discussion in Testi et al. 2001).

Using the $\mathrm{CO}(J=3-2)$ detection we can test the hypothesis of a small, optically thick disk. The mean brightness temperature of the $\mathrm{CO}(J=3-2)$ in our $\sim 00^{\prime \prime} .5 \mathrm{FWHM}$ beam is $\sim 8.3 \mathrm{~K}$. As the gas in the disk is primarily heated by radiation from the central BD, its temperature cannot be very high, i.e., $\lesssim 30-40 \mathrm{~K}$, which in turn means that the beam filling factor of the disk cannot be very small. Under the assumptions of optically thick gas emission and face-on geometry, this argument sets a lower limit of about $15 \mathrm{AU}$ for the disk radius. For example, if the disk radius was $5 \mathrm{AU}$ the beam filling factor would be $<2.3 \%$, the exact value depending on the disk inclination. This would correspond to a gas temperature $>400 \mathrm{~K}$, which is much higher than expected for a BD disk.

The $\beta$-values constrained by our analysis for these larger disks, i.e., $\beta \approx 0.4-0.6$, correspond to dust opacities values of $\kappa_{v, \lambda=0.89 \mathrm{~mm}}^{\text {dust }}=1.5-4.5 \mathrm{~cm}^{-2} \mathrm{~g}^{-1}$ (see also Ricci et al. 2010a). Given the measured flux densities, we derive estimates for the dust mass of $\sim 2-6 \times 10^{-6} M_{\odot}$ or $\sim 2-6 \times 10^{-4} M_{\odot}$ assuming an interstellar medium (ISM)-like gas-to-dust mass ratio of 100. These values correspond to a disk which contains $\sim 0.3 \%-1 \%$ of the mass of the BD+disk system and are consistent with the distribution of values for more massive $\mathrm{T}$ Tauri systems (Williams \& Cieza 2011).

\subsection{Constraints on the Physics of Dust Evolution in Gas-rich Disks}

The $\beta$-values needed for disks larger than 5 AU are significantly lower than the values constrained for the ISM $(\approx 1.6-1.8$; see Draine 2003) and are instead consistent with the values derived for disks around PMS stars ( $\leqslant 1$, e.g., Natta et al. 2007; Ricci et al. 2010a). So far, the only plausible hypothesis proposed to explain these values of $\beta$ is that most of the thermal emission observed in the sub-mm is coming from mm-sized grains, or larger, from the disk's outer regions (Draine 2006; Ricci et al. 2012).

State-of-the-art models of dust evolution in disks can calculate the size-dependent evolution of solids accounting for a variety of different mechanisms, e.g., coagulation, fragmentation, and radial migration (Brauer et al. 2008; Birnstiel et al. 2010a). Sub-mm observations providing information on the grain-size distribution of dust in disks around PMS stars have been used to test the predictions from these models (Birnstiel et al. 2010b; Pinilla et al. 2012). In these models, the efficiency for a disk to grow solids critically depends on its physical conditions. If compared with more massive disks around PMS stars, these models predict that at the conditions of BD disks (e.g., lower densities, higher relative velocities due to radial drift) the growth to $\mathrm{mm}$ sized grains should be much less efficient. Furthermore, in BD disks inward radial drift of $\sim$ mm-sized grains is expected to be faster than in T Tauri disks by a factor of a few. Therefore, the discovery of mm-sized grains in the outer regions of BD disks, as suggested in the case of $\rho$-Oph 102, severely challenges models of the early evolution of solids. Possible ways to explain the presence of mm-sized grains in the outer regions of young BD disks are discussed in P. Pinilla et al. (2012, in preparation). These include very low levels of turbulence in the disk that decrease the collisional speed between solids, and require the 
action of some physical mechanisms that can efficiently halt the inward radial migration of these particles, e.g., gravitational clumping favored by the magnetic field in the disk (Johansen et al. 2007).

\section{SUMMARY}

We presented ALMA observations of the young BD $\rho$-Oph 102 at about $0.89 \mathrm{~mm}$ and $3.2 \mathrm{~mm}$. We reported the detection of the disk surrounding $\rho$-Oph 102 in both the dust continuum bands and in $\operatorname{CO}(J=3-2)$. Dust continuum emission is spatially unresolved, and this provides an upper limit on the radius of the dusty disk of $\sim 40 \mathrm{AU}$. We derived a disk mass of $\sim 0.3 \%-1 \%$ of the mass of the BD, similar to the typical values found for disks around PMS stars. The detection of $\mathrm{CO}$ molecular gas emission at the location of the BD indicates a gas-rich disk as is typically found for disks surrounding more massive young stars. We measured a spectral index of the continuum emission $\alpha_{0.89-3.2 \mathrm{~mm}}=2.29 \pm 0.16$, which is significantly lower than the value found in the ISM. By modeling the dust emission and using the properties of the CO molecular gas emission, we constrained the variation of the dust opacity with frequency, which is a diagnostic of grain growth in the disk. Our result indicates that $\mathrm{mm}$-sized grains are found not only in the outer regions of $\mathrm{T}$ Tauri disks, but also in the low-density environments of BD disks, and this challenges our current understanding of dust evolution in gas-rich disks.

We thank P. Pinilla, T. Birnstiel, and J. Carpenter for helpful comments, and E. van Kampen and ESO ARC for technical support. L.R. acknowledges support from the ESO Scientific Visitor Programme. I.G. is supported by the Spanish MINECO grant AYA2011-30228-C03-01 (co-funded with FEDER fund). This Letter makes use of the following ALMA data: ADS/JAO.ALMA\#2011.0.00259.S. ALMA is a partnership of ESO (representing its member states), NSF (USA), and NINS (Japan), together with NRC (Canada) and NSC and ASIAA (Taiwan), in cooperation with the Republic of Chile. The Joint ALMA Observatory is operated by ESO, AUI/NRAO, and NAOJ. The National Radio Astronomy Observatory is a facility of the National Science Foundation operated under cooperative agreement by Associated Universities, Inc.

\section{REFERENCES}

Andrews, S. M., Wilner, D. J., Hughes, A. M., Qi, C., \& Dullemond, C. P. 2010, ApJ, 723, 1241

Apai, D., Pascucci, I., Bouwman, J., et al. 2005, Science, 310, 834
Beckwith, S. V. W., \& Sargent, A. I. 1991, ApJ, 381, 250

Birnstiel, T., Dullemond, C. P., \& Brauer, F. 2010a, A\&A, 513, 79

Birnstiel, T., Ricci, L., Trotta, F., et al. 2010b, A\&A, 516, L14

Bontemps, S., André, P., Kaas, A. A., et al. 2001, A\&A, 372, 173

Bouy, H., Huélamo, N., Pinte, C., et al. 2008, A\&A, 486, 877

Brauer, F., Dullemond, C. P., \& Henning, Th. 2008, A\&A, 480, 859

Chauvin, G., Lagrange, A. M., \& Dumas, Ch. 2005, A\&A, 438, L25

Chiang, E., \& Goldreich, P. 1997, ApJ, 490, 368

Draine, B. T. 2003, ARA\&A, 41, 241

Draine, B. T. 2006, ApJ, 636, 1114

Dullemond, C. P., Dominik, C., \& Natta, A. 2001, ApJ, 560, 957

Guilloteau, S., Dutrey, A., Pitu, V., \& Boehler, Y. 2011, A\&A, 529, 105

Ho, P. T. P., Moran, J. M., \& Lo, K. Y. 2004, ApJ, 616, L1

Isella, A., Carpenter, J. M., \& Sargent, A. I. 2009, ApJ, 701, 260

Jayawardhana, R., Mohanty, S., \& Basri, G. 2003, ApJ, 592, 282

Johansen, A., Oishi, J. S., Mac Low, M.-M., et al. 2007, Nature, 448,1022

Lodato, G., Delgado-Donate, E., \& Clarke, C. J. 2005, MNRAS, 364, L91

Lombardi, M., Lada, C. J., \& Alves, J. 2008, A\&A, 480, 785

Matsuo, T., Shibai, H., Ootsubo, T., \& Tamura, M. 2007, ApJ, 662, 1282

McMullin, J. P., Waters, B., Schiebel, D., Young, W., \& Golap, K. 2007, in ASP Conf. Ser. 376, Astronomical Data Analysis Software and Systems XVI, ed. R. A. Shaw, F. Hill, \& D. J. Bell (San Francisco, CA: ASP), 127

Mohanty, S., Greaves, J., Mortlock, D., et al. 2012, ApJ, submitted

Natta, A., \& Testi, L. 2001, A\&A, 376, L22

Natta, A., Testi, L., Calvet, N., et al. 2007, in Protostars and Planets V, ed. B. Reipurth, D. Jewitt, \& K. Keil (Tucson, AZ: Univ. Arizona Press), 767

Natta, A., Testi, L., Comern, F., et al. 2002, A\&A, 393, 597

Natta, A., Testi, L., Neri, R., Shepherd, D. S., \& Wilner, D. J. 2004, A\&A, 416, 179

Phan-Bao, N., Riaz, B., Lee, C.-F., et al. 2008, ApJ, 689, 141

Pinilla, P., Birnstiel, T., Ricci, L., et al. 2012, A\&A, 538, 114

Pollack, J. B., Hollenbach, D., Beckwith, S., et al. 1994, ApJ, 421, 615

Ricci, L., Trotta, F., Testi, L., et al. 2012, A\&A, 540, 6

Ricci, L., Testi, L., Natta, A., et al. 2010a, A\&A, 512, 15

Ricci, L., Testi, L., Natta, A., \& Brooks, K. J. 2010b, A\&A, 521, 66

Rodmann, J., Henning, Th., Chandler, C. J., Mundy, L. G., \& Wilner, D. J. 2006, A\&A, 446, 211

Scholz, A., \& Jayawardhana, R. 2008, ApJ, 672, L49

Scholz, A., Jayawardhana, R., \& Wood, K. 2006, ApJ, 645, 1498

Skemer, A. J., Close, L. M., Szucs, L., et al. 2011, ApJ, 732, 107

Sterzik, M. F., Pascucci, I., Apai, D., van der Bliek, N., \& Dullemond, C. P. 2004, A\&A, 427, 245

Testi, L., Natta, A., Oliva, E., et al. 2002, ApJ, 571, L155

Testi, L., Natta, A., Shepherd, D. S., \& Wilner, D. J. 2001, ApJ, 554,1087

Todorov, K., Luhman, K. L., \& McLeod, K. K. 2010, ApJ, 714, L84

Ubach, C., Maddison, S. T., Wright, C. M., et al. 2012, MNRAS, 425, 3137

Warren, S. G. 1984, Appl. Opt., 23, 1206

Weingartner, J. C., \& Draine, B. T. 2001, ApJ, 548, 296

Whelan, E., Ray, T., Bacciotti, F., et al. 2005, Nature, 435, 652

Wilking, B. A., Gagn, M., \& Allen, L. E. 2008, in Handbook of Star Forming Regions, Volume II: The Southern Sky, ed. B. Reipurth (San Francisco, CA: ASP), 351

Williams, J. P., \& Cieza, L. A. 2011, ARA\&A, 49, 67

Zubko, V. G., Mennella, V., Colangeli, L., \& Bussoletti, E. 1996, MNRAS, 282, 1321 